\title{
Mouse Model of Subarachnoid Hemorrhage: Technical Note on the Filament Perforation Model
}

\author{
Muroi, Carl ; Marbacher, Serge ; Fandino, Javier ; Keller, Emanuela ; Katsunori, Iwasaki ; Kenichi, \\ Mishima
}

\begin{abstract}
Experiments using genetically engineered mice are regarded as indispensable to gaining a better understanding of the molecular pathophysiology in neuronal injury after subarachnoid hemorrhage (SAH). Therefore, mouse SAH models are becoming increasingly important. The circle of Willis perforation (cWp) model is the most frequently used mouse SAH model. We report and discuss the technical surgical approach, results, and difficulties associated with the cWp model, with reference to the existing literature. Our results largely confirmed previously published results. This model may be the first choice at present, because important pathologies can be reproduced in this model and most findings in the literature are based on it.
\end{abstract}

DOI: https://doi.org/10.1007/978-3-319-04981-6_54

Posted at the Zurich Open Repository and Archive, University of Zurich

ZORA URL: https://doi.org/10.5167/uzh-115809

Book Section

Accepted Version

Originally published at:

Muroi, Carl; Marbacher, Serge; Fandino, Javier; Keller, Emanuela; Katsunori, Iwasaki; Kenichi, Mishima (2015). Mouse Model of Subarachnoid Hemorrhage: Technical Note on the Filament Perforation Model. In: Fandino, Javier; Marbacher, Serge; Fathi, Ali-Reza; Muroi, Carl; Keller, Emanuela. Neurovascular Events After Subarachnoid Hemorrhage. Cham: Springer, 315-320.

DOI: https://doi.org/10.1007/978-3-319-04981-6_54 


\title{
Mouse Model of Subarachnoid Hemorrhage: Technical Note on the Filament Perforation Model
}

\author{
Carl Muroi, Masayuki Fujioka, Serge Marbacher, Javier Fandino, Emanuela Keller, Katsunori Iwasaki, and Kenichi \\ Mishima
}

\author{
C. Muroi, MD, Department of Neuropharmacology, Faculty of Pharmaceutical Sciences, Fukuoka University, Fukuoka, Japan \\ Department of Neurosurgery, Kantonsspital Aarau, Tellstrasse, CH-5001, Aarau, Switzerland \\ Neurocritical Care Unit, Department of Neurosurgery, University Hospital Zurich, Zurich, Switzerland \\ Department of Intensive Care Medicine, University and University Hospital Bern, Bern, Switzerland e-mail: carl.muroi@ksa.ch
}

M. Fujioka, K. Iwasaki ,K. Mishima Department of Neuropharmacology, Faculty of Pharmaceutical Sciences, Fukuoka University, Fukuoka, Japan

J. Fandino, MD,S. Marbacher, MD, MSc Department of Neurosurgery, Kantonsspital Aarau, Aarau, Switzerland

E. Keller, MD, Neurocritical Care Unit, Department of Neurosurgery, University Hospital Zurich, Zurich, Switzerland e-mail: emanuela.keller@usz.ch

\begin{abstract}
Experiments using genetically engineered mice are regarded as indispensable to gaining a better understanding of the molecular pathophysiology in neuronal injury after subarachnoid hemorrhage (SAH). Therefore, mouse SAH models are becoming increasingly important. The circle of Willis perforation $(\mathrm{cWp})$ model is the most frequently used mouse $\mathrm{SAH}$ model. We report and discuss the technical surgical approach, results, and difficulties associated with the cWp model, with reference to the existing literature. Our results largely confirmed previously published results. This model may be the first choice at present, because important pathologies can be reproduced in this model and most findings in the literature are based on it.
\end{abstract}

\section{Keywords:}

Subarachnoid hemorrhage - Mouse - Mouse model - Vasospasm - Early brain injury

\section{Introduction}

Neuronal injury remains a challenging issue and is associated with a poor outcome after aneurysmal subarachnoid hemorrhage (SAH). Neuronal injury might be cerebral vasospasm (CVS) dependent or independent [16]. A variety of factors at the molecular level have been implicated in the pathogenesis of neuronal injury and/or CVS. These include cytokines and chemokines, leukocyte and platelet adhesion molecules, transcription factors, and upregulated receptors [3, $15,16]$. In this context, experiments using genetically engineered animals might be regarded as indispensable to obtain a better under- standing of the molecular pathophysiology. The number of publications using mouse SAH models has been rapidly increasing during the past few years (Fig. 1a). Reviewing the literature up to 2012, the circle of Willis perforation (cWp) model is the most frequently used model (Fig. 1b). However, reports providing a detailed stepby-step surgical guide for the cWp model were not found. In this brief anecdotal report, we describe the detailed technical surgical approach and summarize our experience and observations. 


\section{Material and Methods}

\section{Animals}

Experiences and observations presented are based on experi- ments performed using ddY (10-12 weeks, 21-26 g, Kyudo Co Ltd., Japan) as well as C57BL/6N (10-12 weeks, 21-26 g, Kyudo Co Ltd., Japan) mice.

Fig. 1 (a) Cumulative number of publications with mouse SAH models, listed by year of publication.

(b) Diagram showing the shares of the models in \%

a

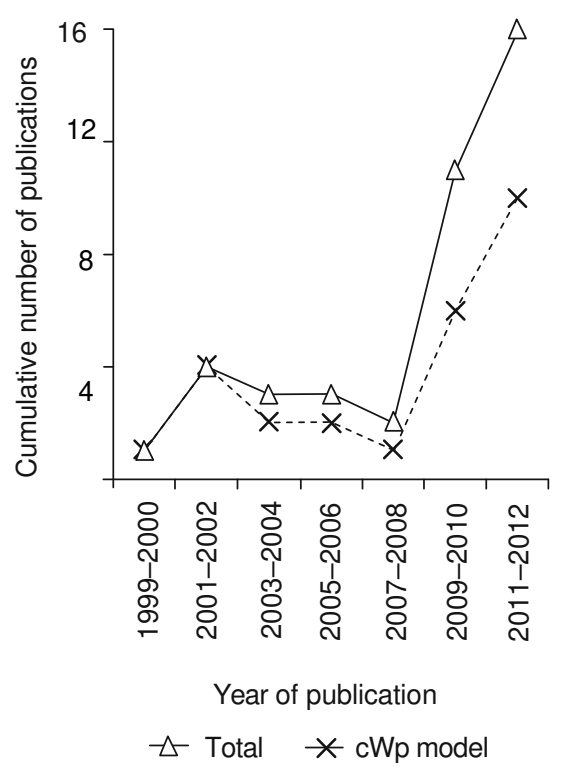

$\triangle$ Total $\quad *$ cWp mode b

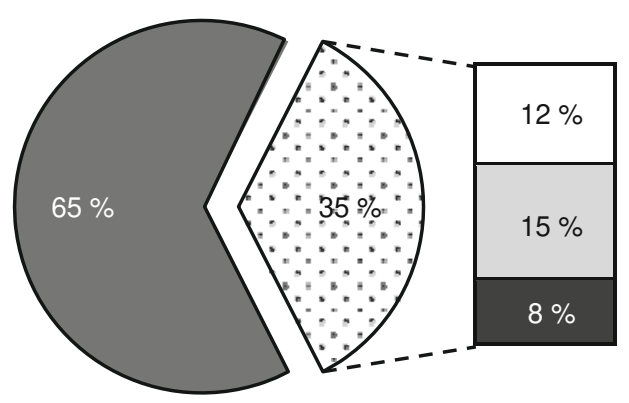

$\square$ cWp model

$\square$ Prechiasmatic injection model

$\square$ Cisterna magna injection model

$\square$ Vessel transsection model

\section{Perioperative Management}

Standard microsurgical instruments including a bipolar forceps were used. The operating microscope had a 7- to 45-fold magnification (Arms Systems Co. Ltd., Japan). Mice were anesthetized with isoflurane (Escain, Mylan Co. Ltd., Japan): 5 $\%$ induction, $1.5 \%$ maintenance, using a facemask. A thermostatically regulated, feedback-controlled heating pad (NSTC10, Neuroscience Inc., Japan) was used to maintain body temperature at $37.5^{\circ} \mathrm{C}$. Intracranial pressure was measured using a fiber-optic micro pressure transducer (Samba Sensors AB, Goteborg, Sweden). In prone position, the tip of the sensor was placed on the left scull base. 


\section{Surgical Procedure}

The mice were placed in a supine position. A midline incision was made in the neck. The underlying subcutaneous tissue was separated in a blunt fashion and the submaxillary glands were gently pushed aside. A triangle formed by the sternohyoid/ omohyoid, sternocleidomastoid, and posterior belly of the digastric muscle was visualized (Fig. 2a). By dissecting the triangle, the neurovascular bundle consisting of the common carotid artery (CCA), vagal nerve, and internal jugular vein was visualized in the depth (Fig. 2b). The CCA was isolated, marked, and gently lifted with a holding thread. The CCA was traced cranially until the bifurcation. At the bifurcation, the occipital artery (OA) - the first branch of the external carotid artery (ECA) - could be seen beside the internal carotid artery (ICA). The ECA was traced in a cranial direction after the digastric muscle was pushed later- ally (Fig. 2b). The ECA was traced until its further branch- ing, preserving the ECA segment as long as possible. After a ligation, the ECA was coagulated and cut. Then, the OA was sacrificed. Afterwards, the ICA, which runs in an inferior- medial direction (point of view), was traced. The removal of the carotid body might be necessary because it might hamper the view. Next, the pterygopalatine artery (PPA) arising from the extracranial section of the ICA was identified (Fig. 2b). The tiny branches from the glossopharyngeal nerve lying on the ICA/PPA bifurcation were gently pushed cranially to gain an adequate view. Once the paths of the ICA and PPA were visible, the PPA was sacrificed (Fig. 2c). If the PPA was not sacrificed, the filament tended to take a via falsa toward the PPA instead of the ICA. A ligation was prepared on the most proximal part of the ECA stump. Mini clips (Roboz Surgical Instrument Co., Inc., USA) were placed at the ICA and CCA. An arteriotomy was performed on the distal part of the ECA stump (Fig. 2c). A 5-0 nylon filament (0.1-mm diameter, Ethilon, Ethicon Inc., USA) was pushed through the arteriotomy toward the ICA (Fig. 2d) until the tip reached the occluded part by the distal clip. One might consider rotating the ECA stump in the inferior direction before inserting the filament, so that the path for the filament would be straight. However, the tip of the filament can get stuck at the bifurcation while it is being advanced. Therefore, we inserted the filament at a more "physiological" angle $\leq 90^{\circ}$ (Fig. 2d). Once the tip overcame the bifurcation, the stump with the filament was rotated inferiorly (Fig. 2e). The clips were removed and the filament was pushed further cranially. After removal of the clips, retrograde bleeding might occur. Gentle tightening of the prepared ligation avoids this. This inconvenience might be further avoided if the proximal clip was left in place and removed at the end of the procedure, i.e., after the perforation and complete withdrawal of the filament and ligation. However, accumulated experience revealed that the latter maneuver decreases the degree of success and volume of SAH, possibly because of rapid activation of the coagulation cascade. Therefore, we recommend the procedure as shown in Fig. $2 \mathrm{~g}$. The filament was gently pushed forward $(\sim 5 \mathrm{~mm})$ until some resistance was felt. The filament was pushed an additional $\sim 1 \mathrm{~mm}$ further to perforate the vessel. The filament was withdrawn quickly and the prepared ligature tightened (Fig. 2f). After ensuring hemostasis, the wound was adapted and sutured. 
Fig. 2 Step-by-step illustration of the cWp model. (a) The triangle formed by the sternohyoid (sh)/omohyoid (oh),

sternocleidomastoid (st), and posterior belly of the digastric muscle $(d p)$ is shown. tr trachea. (b) the neurovascular bundle including the common carotid artery $(c c a)$ and internal jugular vein (ijv) is visualized. eca external carotid artery, ica internal carotid artery, oa occipital artery, $p p a$ pterygopalatine artery, $x i i$ hypoglossal nerve. (c) A stump of the eca is created. The oa and ppa are sacrificed. The Arrowheads indicate the point where the clips are placed. The arrow indicateds the location of the arteriotomy. (d) The filament ( fil) is pushed through the arteriotomy toward the ica. Note the phsiological angle between eca and ica/cca. tip tip of the filament. (e) The stump with the filament is rotated inferiorly once the tip overcome the bifurcation.

(f) After perforation, the filament is withdrawn quickly and the prepared ligature tightened
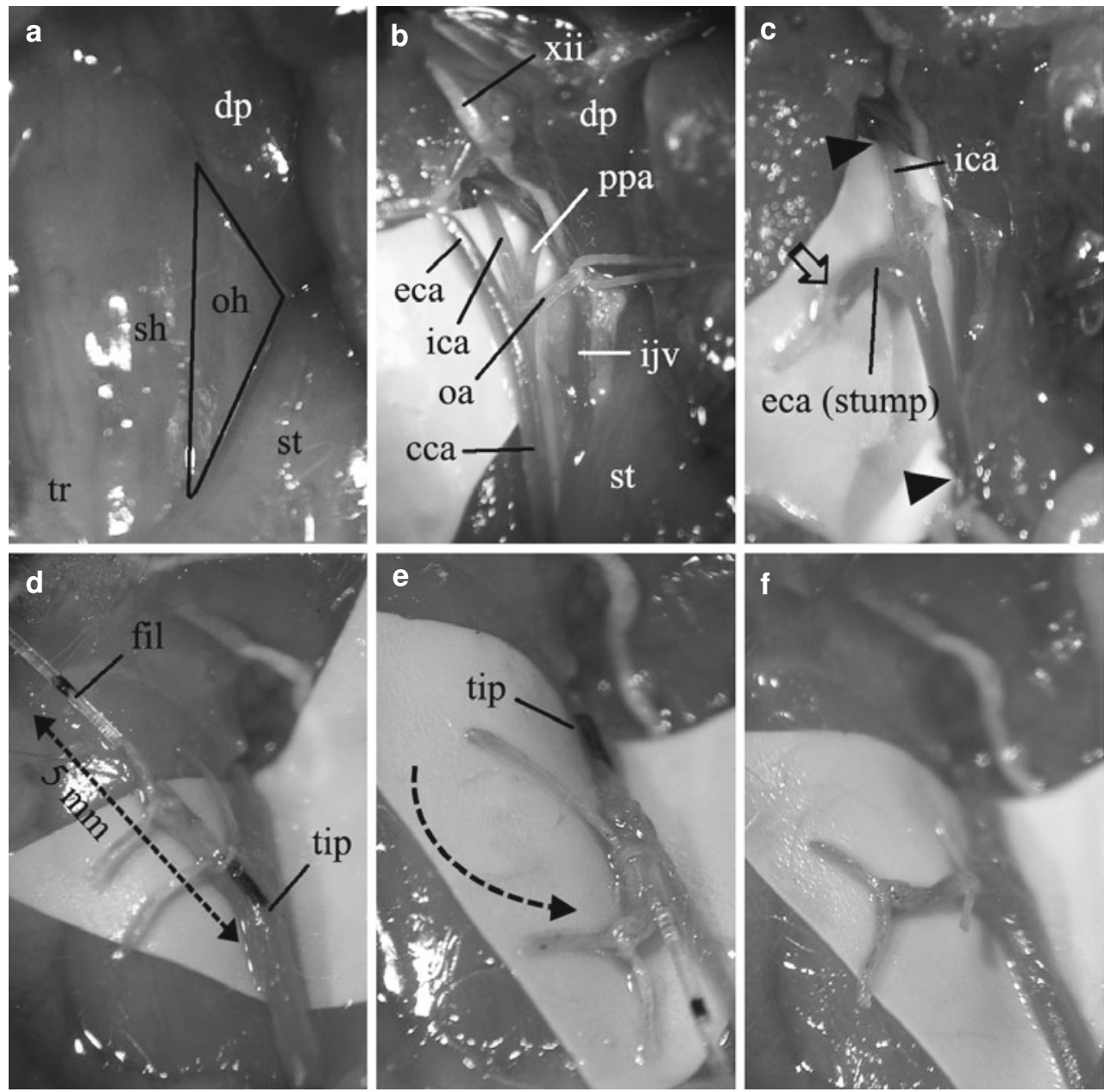

g
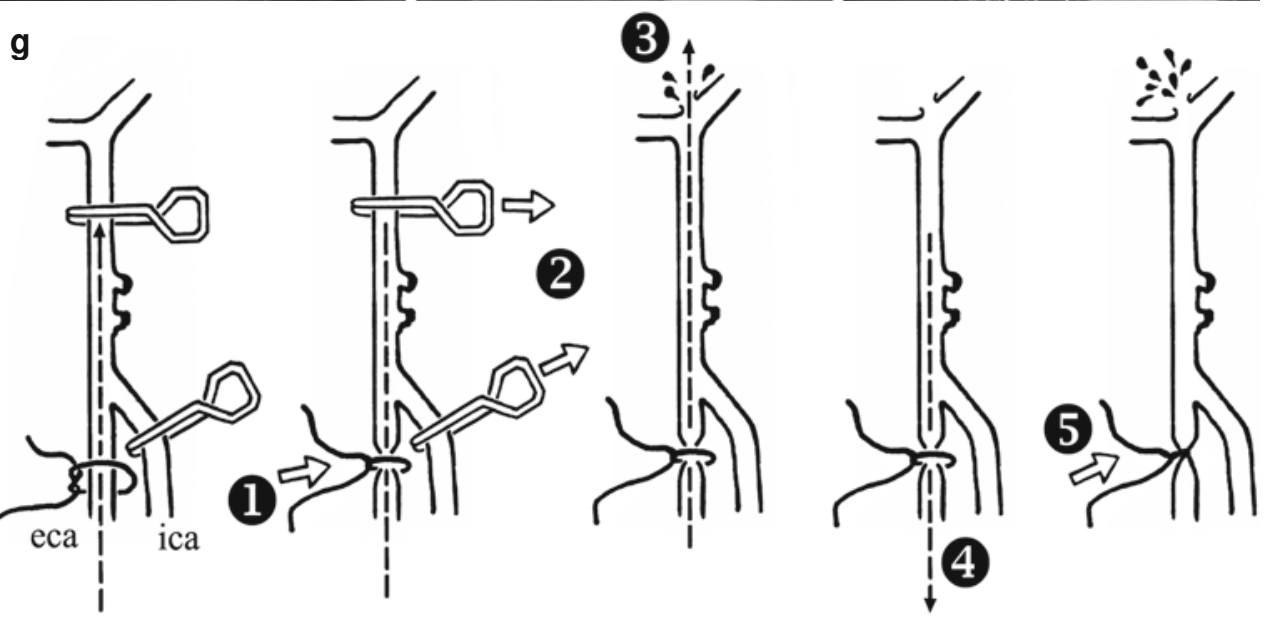


\section{Evaluation of SAH and Histological Examinations}

To evaluate the degree of hemorrhage, animals were killed at day 0, 1, and 2 after SAH. The brains were quickly removed and photographed under the operating microscope. For histological examination, euthanasia was performed by transcardial perfusionfixation at day 2 after $\mathrm{SAH}$. The brains were removed, postfixed and paraffin embedded. The blocks were sliced into 7- $\mu \mathrm{m}$ sections using a microtome (Leitz 1512 Microtome, Wetzlar Germany). Hematoxylin and eosin (HE) staining was performed for overview and CVS identification.

For the assessment of neuronal injury, Fluoro-Jade C (FJC) staining (Merck Millipore, USA) was performed as previously described [21]. To evaluate the occurrence of apoptotic neurons, terminal deoxynucleotidyl transferase dUTP nick labeling (TUNEL) staining was performed (Roche Diagnostics, Germany). The numbers of FJC- and TUNELpositive cells were determined in randomly selected regions of interest in the hippocampal region and cerebral cortex. Because microthrombosis has been discussed more recently as an additional explanation for neuronal injury after SAH, we investigated the occurrence of microthrombosis by immunohistochemistry (sheep anti-fibrinogen anti- body; LifeSpan, BioSciences, USA).

\section{Results}

\section{General Observations}

The perforation caused a sharp increase in ICP. After withdrawal of the filament, the full reperfusion through the ICA caused a further increase in ICP, reflecting a full-scale SAH. We could clearly observe brief decreases in the respiration and pulse frequency in this phase, presumably reflecting a Cushing reflex, as in previous reports [4, 8]. The distance until vessel perforation was achieved varied by 1-3 $\mathrm{mm}$ among the cases. In our experience, this distance cannot be standardized by any means. The distance might vary 1 2 $\mathrm{mm}$ depending on the site of the arteriotomy, or on several other factors, such as the positioning of the animal's head. Therefore, ICP monitoring is probably the method of choice to confirm a successful induction of SAH [4]. However, with accumulating experience, one might success- fully perform it without the need for monitoring, because animals clearly showed a Cushing reflex.

The presence of subarachnoid blood could be verified at all examined time points. The volume of subarachnoid blood varied among the cases. The amount of blood decreased gradually over time (Fig. 3a). Closer inspection of the ventral surface of the brains revealed that the filament perforated the circle of Willis in the vicinity of the ICA/posterior communicating artery (PcomA) bifurcation. In the literature, the majority of reports claimed to have caused a perforation in the vicinity of the anterior cerebral artery (ACA) $[2,5,6,8,10-14,17,20,22-24]$.

Taking the anatomical considerations and the stiffness of the filament into account, the ability to achieve a perforation at the ACA might be considered somewhat doubtful. However, because successful SAH could be induced, the exact location of the perforation might be irrelevant.

The surgical procedure is technically challenging because it requires a meticulous microvascular technique. At the outset, our success rate was approximately $50 \%$, with a high intraoperative morality (surgery-related death before SAH induction) of $25 \%$. After accumulating experience (20 30 cases), the success rate increased to $>90 \%$ and intraoperative mortality decreased to $<5 \%$. Overall SAH-related mortality was $22 \%$ in our series. In the literature, a mortality rate of 10 to $30 \%$ within the first 3 days seems to be a reliable figure for the cWp model $[2,5,6,8,10-14$, $17,20,22-24]$. 


\section{Histological Examinations}

Presence of blood could be histologically verified (Fig. 3b). Morphological signs of CVS could be clearly seen in animals subjected to SAH (Fig. 3b). Degenerative neurons were visible in the ipsilateral cortex (parietal and temporal) and hippocampus. Apoptotic cells, which appeared to be neurons, were found in the ipsilateral cortex on day 2 and 3 after SAH (Fig. 3c). Presence of microthrombi could be observed in animals subjected to SAH (Fig. 3c).

a
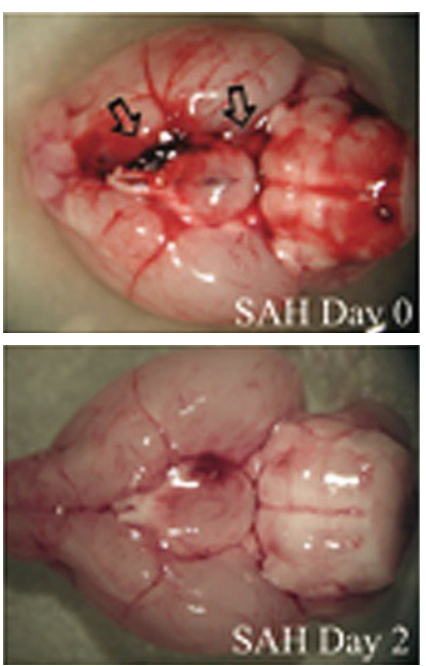

b
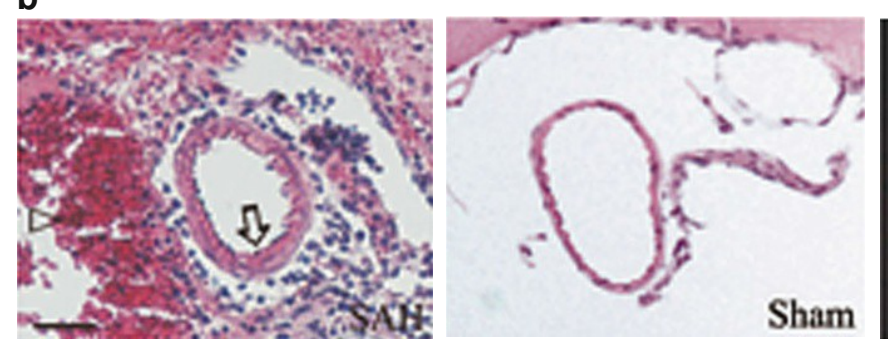

Sham
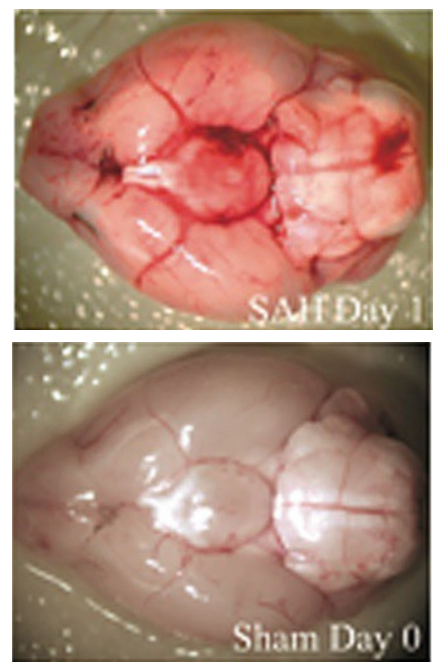

C
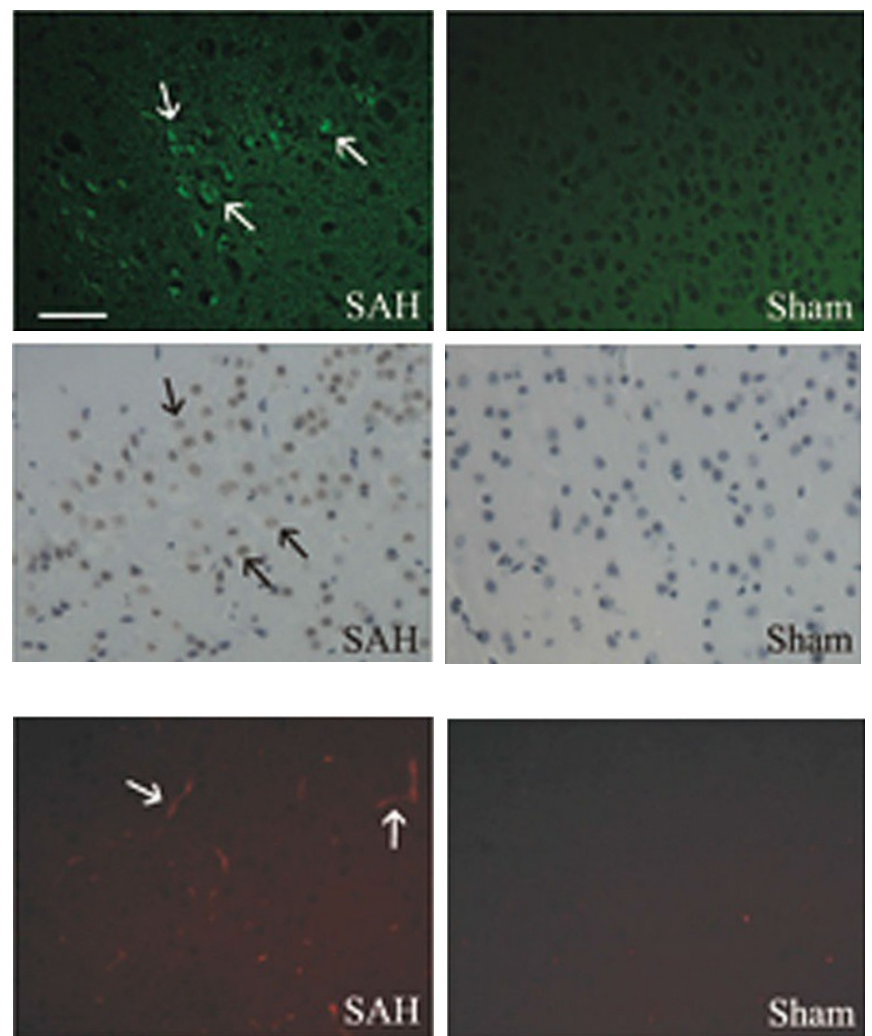

Fig. 3 (a) Representative brain specimens. Subarachnoid blood in the basal cisterns is clearly visible at day 0 (arrows). The amount of blood decreased with time. No SAH was seen in sham animals.

(b) Representative image sections of the distal ICA showing morphological signs of CVS with thickened vessel wall in SAH (arrow). Note the subarachnoid blood (arrowhead). Bar: $50 \mu \mathrm{m}$.

(c) Upper row: FJC- positive neurons after SAH (arrows). Middle row: TUNEL-positive cells after SAH (arrows).Bottom row: Fibrinogen-positive microthrombi after SAH (arrows). Bar: $100 \mu \mathrm{m}$ 


\section{Discussion}

We successfully reconstructed and established the cWp SAH model, showing important pathologies such as vessel rupture, ICP increase, Cushing reflex, microthrombosis, CVS and neuronal injury, hence confirming previous studies. This mouse model was derived from the rat filament perforation model and was reported for the first time by Kamii et al. in 1999 [8]. The first paper dedicated to the model itself was published by Parra et al. in 2002 [17]. Another paper dedicated to the model itself followed in 2010 [4]. In the latter, suitable intraoperative parameters for the controlled and standardized induction of SAH were described.

It is common sense to consider that the initial amount of hemorrhage influences the degree of brain injury. Further, the time period during which erythrocytes break down and blood disappears from the CSF compartment closely mirrors the onset and resolution of CVS. The compartmental inflammatory response, which might contribute to CVSdependent or -independent neuronal injury, is triggered by erythrocyte breakdown and also by hemoglobin release [15]. Therefore, the size of the hematoma and the kinetics of its resorption are of interest. It is well accepted that in smaller animals (e.g., lissencephalic rodents), the clearance of the clot is more rapid than in larger animals (e.g., gyrenecephalic primates) [9]. However, detailed information about the rate of clot clearance in mouse SAH is rare in the literature. A detailed description is available for the cisterna magna injection model. One hour after SAH, a large diffuse blood clot was described in the subarachnoid space; on day 1 , the clot was thinner, but still clearly visible around the major cerebral arteries; on day 3 or 4 , the cisternal blood clot was essentially absent [9]. Our observations indicated a similar kinetic in hematoma resorption. The latter might explain the early onset of CVS in the mouse SAH model, which, in turn, might compromise the discrimination between the effects of early brain injury and CVS.

At this point, the impossibility of controlling the degree of SAH has to be mentioned again as the most crucial disadvantage of the cWp model, as already discussed in the literature [4, 9]. Advantages and disadvantages of the cWp model com- pared with other models have been discussed in detail else- where $[1,4,10,19]$. In brief, the cWp model is technically (surgically) the most challenging model. Further, differentiation between the effects of SAH per se and effects of intracranial hypertension is not possible [18]. However, the model replicates the human pathophysiology satisfactorily, including vessel rupture and endothelial damage, which might be important features. No model other than the cWp model reflects the latter aspect. Furthermore, the superiority of the $\mathrm{cWp}$ model to reproduce early platelet-leukocyteendothelial cell interactions after SAH has been described more recently [7].

\section{Conclusions}

Mice models are becoming increasingly popular, as better understanding of SAH-induced brain injury at the molecular level, using genetically engineered animals, is needed. The cWp model is the most frequently used SAH model in mice. The model replicates the important pathophysiology satisfactorily.

Acknowledgments CM has been supported by a personal research grant from the Swiss Foundation for Grants in Biology and Medicine and F. Hoffmann-La Roche Ltd., Basel, Switzerland

Conflict of Interest Statement We declare that we have no conflict of interest. 


\section{References}

1. Altay T, Smithason S, Volokh N, Rasmussen PA, Ransohoff RM, Provencio JJ (2009) A novel method for subarachnoid hemorrhage to induce vasospasm in mice. J Neurosci Methods 183:136-140

2. Borel CO, McKee A, Parra A, Haglund MM, Solan A, Prabhakar V, Sheng H, Warner DS, Niklason L (2003) Possible role for vas- cular cell proliferation in cerebral vasospasm after subarachnoid hemorrhage. Stroke 34:427-433

3. Chaichana KL, Pradilla G, Huang J, Tamargo RJ (2010) Role of inflammation (leukocyte-endothelial cell interactions) in vaso- spasm after subarachnoid hemorrhage. World Neurosurg 73:22-41

4. Feiler S, Friedrich B, Scholler K, Thal SC, Plesnila N (2010) Standardized induction of subarachnoid hemorrhage in mice by intracranial pressure monitoring. J Neurosci Methods 190:164-170

5. Gao J, Wang H, Sheng H, Lynch JR, Warner DS, Durham L, Vitek MP, Laskowitz DT (2006) A novel apoE-derived therapeutic reduces vasospasm and improves outcome in a murine model of subarachnoid hemorrhage. Neurocrit Care 4:25-31

6. Han BH, Vellimana AK, Zhou ML, Milner E, Zipfel GJ (2012) Phosphodiesterase 5 inhibition attenuates cerebral vasospasm and improves functional recovery after experimental subarachnoid hemorrhage. Neurosurgery 70:178-186

7. Ishikawa M, Kusaka G, Yamaguchi N, Sekizuka E, Nakadate H, Minamitani H, Shinoda S, Watanabe E (2009) Platelet and leuko- cyte adhesion in the microvasculature at the cerebral surface imme- diately after subarachnoid hemorrhage. Neurosurgery 64:546-553, discussion 553-544

8. Kamii H, Kato I, Kinouchi H, Chan PH, Epstein CJ, Akabane A, Okamoto H, Yoshimoto T (1999) Amelioration of vasospasm after subarachnoid hemorrhage in transgenic mice overexpressing CuZn-superoxide dismutase. Stroke 30:867-871, discussion 872

9. Lin CL, Calisaneller T, Ukita N, Dumont AS, Kassell NF, Lee KS (2003) A murine model of subarachnoid hemorrhage-induced cerebral vasospasm. J Neurosci Methods 123:89-97

10. Liu S, Tang J, Ostrowski RP, Titova E, Monroe C, Chen W, Lo W, Martin R, Zhang JH (2007) Oxidative stress after subarachnoid hem- orrhage in gp91phox knockout mice. Can J Neurol Sci 34:356-361

11. McGirt MJ, Lynch JR, Parra A, Sheng H, Pearlstein RD, Laskowitz DT, Pelligrino DA, Warner DS (2002) Simvastatin increases endo- thelial nitric oxide synthase and ameliorates cerebral vasospasm resulting from subarachnoid hemorrhage. Stroke 33:2950-2956

12. McGirt MJ, Parra A, Sheng H, Higuchi Y, Oury TD, Laskowitz DT, Pearlstein RD, Warner DS (2002) Attenuation of cerebral vasospasm after subarachnoid hemorrhage in mice overexpressing extracellular superoxide dismutase. Stroke 33:2317-2323

13. Mesis RG, Wang H, Lombard FW, Yates R, Vitek MP, Borel CO, Warner DS, Laskowitz DT (2006) Dissociation between vaso- spasm and functional improvement in a murine model of subarach- noid hemorrhage. Neurosurg Focus 21:E4

14. Mino M, Kamii H, Fujimura M, Kondo T, Takasawa S, Okamoto H, Yoshimoto $T$ (2003) Temporal changes of neurogenesis in the mouse hippocampus after experimental subarachnoid hemorrhage. Neurol Res 25:839-845

15. Muroi C, Mink S, Seule M, Bellut D, Fandino J, Keller E (2011) Monitoring of the inflammatory response after aneurysmal subarachnoid haemorrhage in the clinical setting: review of literature and report of preliminary clinical experience. Acta Neurochir Suppl 110:191-196

16. Muroi C, Seule M, Mishima K, Keller E (2012) Novel treatments for vasospasm after subarachnoid hemorrhage. Curr Opin Crit Care 18:119-126

17. Parra A, McGirt MJ, Sheng H, Laskowitz DT, Pearlstein RD, Warner DS (2002) Mouse model of subarachnoid hemorrhage associated cerebral vasospasm: methodological analysis. Neurol Res 24:510-516

18. Sabri M, Ai J, Lakovic K, D'Abbondanza J, Ilodigwe D, Macdonald RL (2012) Mechanisms of microthrombi formation after experi- mental subarachnoid hemorrhage. Neuroscience 224:26-37

19. Sabri M, Jeon H, Ai J, Tariq A, Shang X, Chen G, Macdonald RL (2009) Anterior circulation mouse model of subarachnoid hemor- rhage. Brain Res 1295:179-185

20. Saito A, Kamii H, Kato I, Takasawa S, Kondo T, Chan PH, Okamoto H, Yoshimoto T (2001) Transgenic CuZn-superoxide dismutase inhibits NO synthase induction in experimental sub- arachnoid hemorrhage. Stroke 32:1652-1657

21. Schmued LC, Stowers CC, Scallet AC, Xu L (2005) Fluoro-Jade C results in ultrahigh resolution and contrast labeling of degenerat- ing neurons. Brain Res 1035:24-31

22. Sozen T, Tsuchiyama R, Hasegawa Y, Suzuki H, Jadhav V, Nishizawa S, Zhang JH (2009) Role of interleukin-1beta in early brain injury after subarachnoid hemorrhage in mice. Stroke 40:2519-2525

23. Suzuki H, Sozen T, Hasegawa Y, Chen W, Zhang JH (2009) Caspase-1 inhibitor prevents neurogenic pulmonary edema after subarachnoid hemorrhage in mice. Stroke 40:3872-3875

24. Wakade C, King MD, Laird MD, Alleyne CH Jr, Dhandapani KM (2009) Curcumin attenuates vascular inflammation and cerebral vasospasm after subarachnoid hemorrhage in mice. Antioxid Redox Signal 11:35-45 